\title{
Encyclopedia
}

\section{Type 2 Diabetes Mellitus}

Created by: Vishwanath Venketaraman

Version received: 27 December 2019

Uncontrolled Type 2 diabetes is associated with altered metabolism resulting in microvascular and macrovascular diseases and enhanced susceptibility to Mycobacterium tuberculosis infection.

\section{Type 2 Diabetes Mellitus}

Type 2 Diabetes Mellitus is a prevalent disease throughout the world. The World Health Organization (WHO) reports that an estimated 422 million people all over the world are living with diabetes. The WHO projects that diabetes will be the seventh leading cause of death in 2030 [1]. In the United States, the Center for Disease Control (CDC) reports that 30.3 million people have diabetes, with approximately 23.1 million people diagnosed every year and a staggering 84.1 million Americans in pre-diabetic stage [2]. Tuberculosis (TB) is a serious threat for people living with diabetes. In $2017,20 \%$ of people with TB in the United States also had diabetes. Currently, one third of the world's population is latently infected with Mycobacterium tuberculosis (M. tb) [3]. At this stage, the individual is not infectious; however, latent $M$. tb can be reactivated as a result of granuloma liquefaction due to immunodeficiency or immunocompromisation [4], as in the case with individuals suffering from Type 2 Diabetes Mellitus (T2DM) [5]. Due to the high prevalence of diabetes worldwide, necessary measures must be taken to understand, prevent, and treat T2DM.

Insulin, a peptide hormone produced by beta cells within the pancreas, facilitates the absorption of glucose into cells from blood, in order to maintain proper glucose levels [6]. T2DM is a disease characterized by the inability of the body to produce sufficient insulin, or the development of insulin resistance [7]. As a result, T2DM's state of insufficient insulin production or insulin-resistance can have detrimental complications, including macrovascular diseases such as hypertension, coronary artery disease, heart attacks and strokes; or microvascular diseases such as neuropathy, nephropathy, and cancer [8]. In order to effectively reduce the incidence of T2DM worldwide and prevent concurrent infections, efforts must be undertaken to discern the etiology, to create rationale treatments for symptomatic patients, and to develop preventive measures.

\section{Pathogenesis in Type 2 Diabetes Mellitus}

Diabetes complications resulting from an impaired glucose metabolism have been associated with retinopathy, nephropathy, and polyneuropathy [9]. When present, excess glucose is not oxidized and is shunted to the polyol pathway, consisting of two enzymes—aldose reductase (AR) and sorbitol dehydrogenase (SDH) [10]. AR reduces glucose to sorbitol in the presence of its co-factor, NADPH; SDH, with its co-factor nicotinamide adenine dinucleotide (NAD+), converts sorbitol to fructose-a more potent nonenzymatic glycation agent than glucose. Thus, an increase in glucose flux through the polyol pathway (as happens in T2DM) will decrease the available NADPH and increase the production of Advanced Glycation End products (AGE), leading to oxidative stress in a pathway we discuss later in this review [11]. The SDH oxidation of sorbitol to fructose also causes oxidative stress due to the co-factor conversion of $\mathrm{NAD}$ + to $\mathrm{NADH}$, the substrate for $\mathrm{NADH}$ oxidase which generates reactive oxygen species (ROS) [12]. Elevated sorbitol levels have also been associated with cellular and organ damage by directly depleting myoinositol (MI). This MI deficiency alters the metabolism of phosphoinositides and reduces diacylglycerol (DAG), inositol triphosphate (IP3) and protein kinase $\mathrm{C}(\mathrm{PKC})$ activation, resulting in a reduction in $\mathrm{NA}^{+} / \mathrm{K}^{+} \mathrm{ATPase}$ pump activity. Metabolic pathway derangement causes conductance abnormalities, resulting in neurological complications such as the neuropathy observed in diabetic patients [10]. Additionally, SDH enzyme deficiency in nervous tissues, the kidney, and the lens and retina of the eye lead to the elevation of sorbitol, leading to the development and progression of retinopathy, cataracts and neuropathy observed in diabetic patients [13].

Concomitantly, the cellular antioxidant capacity of Glutathione (GSH) is diminished due to AR activity, which depletes the NADPH required for glutathione reductase (GSR) to recycle glutathione (GSH). A reduction in available NADPH, due to 


\section{Encyclopedia}

the overutilization of the Polyol Pathway, significantly decreases GSH levels and thus limits the ROS-scavenging activity of GSH, and further increases ROS levels [14]. A diabetic mouse model (MKR) vs. control study performed in 2010 revealed sorbitol levels 2.5 times higher in MKR when compared to a control Also found was 1.7 times lower levels of the reduced glutathione (rGSH) in skeletal muscle [15]. Therefore, it is understood that the overutilization of the polyol pathway due to glucose excess is a major source of oxidative stress, by limiting the recycling of GSH via GSR induced by diabetes.

\section{Keywords}

type 2 diabetes; TB,; Co-morbidities; oxidative stress 